\title{
A new characterization of $A_{7}$ and $A_{8}$
}

\author{
Alireza Khalili Asboei, Seyed Sadegh Salehi Amiri, Ali Iranmanesh \\ and Abolfazl Tehranian
}

\begin{abstract}
Let $G$ be a finite group and $\pi_{e}(G)$ be the set of element orders of $G$. Let $k \in \pi_{e}(G)$ and $m_{k}$ be the number of elements of order $k$ in $G$. Set $\operatorname{nse}(G):=\left\{m_{k} \mid k \in \pi_{e}(G)\right\}$. It is proved that $A_{n}$ are uniquely determined by nse $\left(A_{n}\right)$, where $n \in\{4,5,6\}$. In this paper, we prove that if $G$ is a group such that $\operatorname{nse}(G)=\operatorname{nse}\left(A_{n}\right)$ where $n \in\{7,8\}$, then $G \cong A_{n}$.
\end{abstract}

\section{Introduction}

If $n$ is an integer, then we denote by $\pi(n)$ the set of all prime divisors of $n$. Let $G$ be a finite group. Denote by $\pi(G)$ the set of primes $p$ such that $G$ contains an element of order $p$. Also the set of element orders of $G$ is denoted by $\pi_{e}(G)$. A finite group $G$ is called a simple $K_{n}$-group, if $G$ is a simple group with $|\pi(G)|=n$.

Set $m_{i}=m_{i}(G)=\mid\{g \in G \mid$ the order of $g$ is $i\} \mid$. In fact, $m_{i}$ is the number of elements of order $i$ in $G$, and nse $(G):=\left\{m_{i} \mid i \in \pi_{e}(G)\right\}$, the set of sizes of elements with the same order. For the set nse $(G)$, the most important problem is related to Thompson's problem. In 1987, J. G. Thompson posed a very interesting problem related to algebraic number fields as follows. For each finite group $G$ and each integer $d \geq 1$, let $G(d)=\left\{x \in G \mid x^{d}=1\right\}$. Defining $G_{1}$ and $G_{2}$ is of the same order type if, and only if, $\left|G_{1}(d)\right|=\left|G_{2}(d)\right|, d=1$, group.

Key Words: Element order, set of the numbers of elements of the same order, alternating

2010 Mathematics Subject Classification: Primary 20D60.

Received: January, 2012.

Revised: May, 2012.

Accepted: March, 2013. 
$2,3, \cdots$. Suppose $G_{1}$ and $G_{2}$ are of the same order type. If $G_{1}$ is solvable, is $G_{2}$ necessarily solvable?( [10, Problem 12.37])

We know that if groups $G_{1}$ and $G_{2}$ are of the same order type, then $\left|G_{1}\right|=\left|G_{2}\right|$ and nse $\left(G_{1}\right)=\operatorname{nse}\left(G_{2}\right)$. So it is natural to investigate the Thompson's Problem by $|G|$ and nse $(G)$.

In [4], [2], [3] and [1], it is proved that all simple $K_{4}-$ groups, symmetric groups $S_{r}$ where $r$ is prime, sporadic simple groups and $L_{2}(p)$ where $p$ is prime, can be uniquely determined by nse $(G)$ and the order of $G$. In [9] and [8], it is proved that the groups $A_{4}, A_{5}$ and $A_{6}, L_{2}(q)$ for $q \in\{7,8,11,13\}$ are uniquely determined by only nse $(G)$. In [9], the authors gave the following problem:

Problem: Is a group $G$ isomorphic to $A_{n}(n \geq 4)$ if and only if nse $(G)$ $=\operatorname{nse}\left(A_{n}\right)$ ?

In this paper, we give a positive answer to this problem and show that the alternating group $A_{n}$ is characterizable by only nse $(G)$ for $n \in\{7,8\}$. In fact the main theorem of our paper is as follows:

Main Theorem: Let $G$ be a group such that $\operatorname{nse}(G)=\operatorname{nse}\left(A_{n}\right)$, where $n \in\{7$, 8\}. Then $G \cong A_{n}$.

We note that there are finite groups which are not characterizable even by nse $(G)$ and $|G|$. In 1987, Thompson gave an example as follows: Let $G_{1}=$ $\left(C_{2} \times C_{2} \times C_{2} \times C_{2}\right) \rtimes A_{7}$ and $G_{2}=L_{3}(4) \rtimes C_{2}$ be the maximal subgroups of $M_{23}$. Then nse $\left(G_{1}\right)=\operatorname{nse}\left(G_{2}\right)$ and $\left|G_{1}\right|=\left|G_{2}\right|$, but $G_{1} \neq G_{2}$. Throughout this paper, we denote by $\phi$ the Euler totient function. If $G$ is a finite group, then we denote by $P_{q}$ a Sylow $q-\operatorname{subgroup~of~} G$ and $n_{q}(G)$ is the number of Sylow $q$-subgroup of $G$, that is, $n_{q}(G)=\left|\operatorname{Syl}_{q}(G)\right|$. All other notations are standard and we refer to [7], for example.

\section{Main Results}

In this section, for the proof of main theorem, we need the following Lemmas: Lemma 2.1. [5] Let $G$ be a finite group and $m$ be a positive integer dividing $|G|$. If $L_{m}(G)=\left\{g \in G \mid g^{m}=1\right\}$, then $m|| L_{m}(G) \mid$.

Lemma 2.2. [6] Let $G$ be a finite group and $p \in \pi(G)$ be odd. Suppose that $P$ is a Sylow $p$-subgroup of $G$ and $n=p^{s} m$, where $(p, m)=1$. If $P$ is not cyclic and $s>1$, then the number of elements of order $n$ is always a multiple of $p^{s}$.

Lemma 2.3. [9] Let $G$ be a group containing more than two elements. 
Let $k \in \pi_{e}(G)$ and $m_{k}$ be the number of elements of order $k$ in $G$. If $s=$ $\sup \left\{m_{k} \mid k \in \pi_{e}(G)\right\}$ is finite, then $G$ is finite and $|G| \leq s\left(s^{2}-1\right)$.

Let $G$ be a group such that nse $(G)=$ nse $\left(A_{n}\right)$, where $n \in\{7,8\}$. By Lemma 2.3 , we can assume that $G$ is finite. Let $m_{n}$ be the number of elements of order $n$. We note that $m_{n}=k \phi(n)$, where $k$ is the number of cyclic subgroups of order $n$ in $G$. Also we note that if $n>2$, then $\phi(n)$ is even. If $n|| G \mid$, then by Lemma 2.1 and the above notation we have

$$
\left\{\begin{array}{l}
\phi(n) \mid m_{n} \\
n \mid \sum_{d \mid n} m_{d}
\end{array}\right.
$$

In the proof of the main theorem, we often apply $(*)$ and the above comments.

\section{Proof of the Main Theorem}

Let $G$ be a group such that nse $(G)=\operatorname{nse}\left(A_{7}\right)=\{1,105,210,350,504,630,720\}$. First we prove that $\pi(G) \subseteq\{2,3,5,7\}$. Since $105 \in \operatorname{nse}(G)$, it follows that by $(*), 2 \in \pi(G)$ and $m_{2}=105$. Let $2 \neq p \in \pi(G)$. By $(*), p \mid\left(1+m_{p}\right)$ and $(p-1) \mid m_{p}$, which implies that $p \in\{3,5,7,211,631\}$. If $211 \in \pi(G)$, then by $(*), m_{211}=210$. On the other hand, by $(*)$ we conclude that if $422 \in \pi_{e}(G)$, then $m_{422}=210$ or 630 and $422 \mid\left(1+m_{2}+m_{211}+m_{422}\right)$, and hence $422 \mid 526$ or $422 \mid 946$, which is a contradiction. Thus $422 \notin \pi_{e}(G)$. Since $422 \notin \pi_{e}(G)$, the group $P_{211}$ acts fixed point freely on the set of elements of order 2 , and so $\left|P_{211}\right| \mid m_{2}$, which is a contradiction. Hence $211 \notin \pi_{e}(G)$. Similar to the above discussion $631 \notin \pi(G)$.

If 3,5 and $7 \in \pi(G)$, then $m_{3}=350, m_{5}=504$ and $m_{7}=720$, by $(*)$. Also we can see easily that $G$ does not contain any elements of order 35,81 , 64, 125 and 343. Similarly, we can see that if $10,14,15,21 \in \pi_{e}(G)$, then $m_{10}=720, m_{14} \in\{210,504\}, m_{15}=720$ and $m_{21}=504$.

If $2^{a} \times 3^{b} \in \pi_{e}(G)$, then $2^{a} \times 3^{b-1} \mid m_{2^{a} \times 3^{b}}$. Hence $1 \leq a \leq 3$ and $1 \leq b \leq 3$.

If $2^{c} \times 5^{d} \in \pi_{e}(G)$, then $2^{c+1} \times 5^{d-1} \mid m_{2^{c} \times 5^{d}}$. Hence $1 \leq c \leq 3$ and $1 \leq d \leq 2$.

If $2^{e} \times 7^{f} \in \pi_{e}(G)$, then $2^{e} \times 3 \times 7^{f-1} \mid m_{2^{e} \times 7^{f}}$. Hence $1 \leq e \leq 3$ and $1 \leq f \leq 2$.

If $3^{k} \times 5^{h} \in \pi_{e}(G)$, then $2^{3} \times 3^{k-1} \times 5^{h-1} \mid m_{3^{k} \times 5^{h}}$. Hence $1 \leq k \leq 3$ and $1 \leq h \leq 2$.

If $3^{l} \times 7^{m} \in \pi_{e}(G)$, then $2^{2} \times 3^{l} \times 7^{m-1} \mid m_{3^{l} \times 7^{m}}$. Hence $1 \leq l \leq 2$ and $1 \leq m \leq 2$. 
In follow, we show that $\pi(G)$ could not be the sets $\{2\},\{2,3\},\{2,3,7\}$ and $\{2,3,5\}$, and $\pi(G)$ must be equal to $\{2,3,5,7\}$.

Case a. If $\pi(G)=\{2\}$, then $\pi_{e}(G) \subseteq\left\{1,2,2^{2}, 2^{3}, 2^{4}, 2^{5}\right\}$. Since nse $(G)$ has seven elements, this case impossible.

Case b. We know that $2 \in \pi(G)$. We claim that $3 \in \pi(G)$. Suppose that $3 \notin \pi(G)$. If $5,7 \notin \pi(G)$, then by Case a, we get a contradiction. Hence 5 or $7 \in \pi(G)$.

Let $5 \in \pi(G)$. Since $125 \notin \pi_{e}(G), \exp \left(P_{5}\right)=5$ or 25 . If $\exp \left(P_{5}\right)=5$, then by Lemma 2.1, $\left|P_{5}\right| \mid\left(1+m_{5}\right)=505$. Hence $\left|P_{5}\right|=5$. Then $n_{5}=m_{5} / \phi(5)=$ $504 / 4|| G \mid$, a contradiction. If $\exp \left(P_{5}\right)=25$, then $\left|P_{5}\right| \mid\left(1+m_{5}+m_{25}\right)$. Hence $\left|P_{5}\right|=25$ and $n_{5}=m_{25} / 20|| G \mid$. Since $m_{25}=720$, we get a contradiction. Thus $5 \notin \pi(G)$.

Let $7 \in \pi(G)$. Since $7^{3} \notin \pi_{e}(G), \exp \left(P_{7}\right)=7$ or 49 . If $\exp \left(P_{7}\right)=7$, then by Lemma $2.1,\left|P_{7}\right| \mid\left(1+m_{7}\right)=721$. Hence $\left|P_{7}\right|=7$ and $n_{7}=m_{7} / \phi(7)|| G \mid$, which is a contradiction.

If $\exp \left(P_{7}\right)=49$, then $\left|P_{7}\right| \mid\left(1+m_{7}+m_{49}\right)$. Hence $\left|P_{7}\right|=49$. Since $m_{49} \in\{210,504\}, n_{7}=m_{49} / \phi(49)=5$ or 12 . By Sylow's theorem $n_{7}=7 k+1$ for some $k$, since $n_{7}=5$ or 12 , we get a contradiction. Thus $3 \in \pi(G)$.

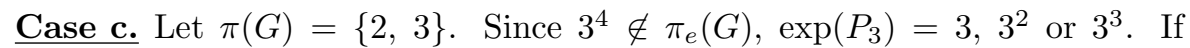
$\underline{\exp \left(P_{3}\right)}=3,\left|P_{3}\right| \mid\left(1+m_{3}\right)=351$, by Lemma 2.1. Thus $\left|P_{3}\right| \mid 3^{3}$. If $\left|P_{3}\right|=3$, then $n_{3}=m_{3} / 2|| G \mid$, a contradiction. If $\left|P_{3}\right|>3$, then since $\exp \left(P_{3}\right)=3$, $\left|\pi_{e}(G)\right| \leq 11$. Therefore $|G|=2^{m} \times 3^{n}=2520+350 k_{1}+504 k_{2}+720 k_{3}+$ $630 k_{4}+210 k_{5}$, where $m, n, k_{1}, k_{2}, k_{3}, k_{4}$ and $k_{5}$ are non-negative integers and $0 \leq k_{1}+k_{2}+k_{3}+k_{4}+k_{5} \leq 4$. It is clear that $|G| \leq 5400$. If $n=2$, then $m=9$. It easy to check that the above equation has no solution. If $n=3$, then $m=7$, arquing as above, the equation has no solution. Therefore $\exp \left(P_{3}\right) \neq 3$.

If $\exp \left(P_{3}\right)=9$, by Lemma $2.1,\left|P_{3}\right| \mid\left(1+m_{3}+m_{9}\right)$. Since $m_{9} \in\{504,630$, $720\},\left|P_{3}\right|=9$. Hence $n_{3}=m_{9} / 6|| G \mid$, a contradiction.

If $\exp \left(P_{3}\right)=27$, then since $m_{27} \in\{504,630,720\},\left|P_{3}\right| \mid 3^{5}$. If $\left|P_{3}\right|=27$, then $n_{9}=m_{27} / 18|| G \mid$, a contradiction. If $\left|P_{3}\right|=81$ or 243 , then by Lemma $2.2,3^{3} \mid m_{27}$, a contradiction.

Case d. Let $\pi(G)=\{2,3,7\}$. Since $7^{3} \notin \pi_{e}(G), \exp \left(P_{7}\right)=7$ or $7^{2}$. If $\exp \left(P_{7}\right)=7$, then $\left|P_{7}\right| \mid\left(1+m_{7}\right)=721$. Hence $\left|P_{7}\right|=7$ and $n_{7}=m_{7} / 6=$ 120||$G \mid$, a contradiction.

If $\exp \left(P_{7}\right)=49$, then $\left|P_{7}\right| \mid\left(1+m_{7}+m_{49}\right)$. Thus $\left|P_{7}\right|=49$ and $n_{7}=m_{49} / 42=5$ or 12 . By Sylow's theorem, we get a contradiction. 
Case e. Let $\pi(G)=\{2,3,5\}$. Since $125 \notin \pi_{e}(G), \exp (G)=5$ or 25 . If $\exp (G)=5$, then $\left|P_{5}\right| \mid\left(1+m_{5}\right)=505$. Hence $\left|P_{5}\right|=5$ and $n_{5}=m_{5} / 4=$ 126||$G \mid$, which is a contradiction.

If $\exp \left(P_{5}\right)=25$, then $\left|P_{5}\right| \mid\left(1+m_{5}+m_{25}\right)=1225$. Hence $\left|P_{5}\right|=25$ and then the group $P_{5}$ is cyclic. Thus $n_{5}=m_{25} / 20=36$. Since a cyclic group of order 25 has 4 elements of order $5, m_{5} \leq 4 \times n_{5}=144$, which is a contradiction.

Case f. Let $\pi(G)=\{2,3,5,7\}$. Since $35 \notin \pi_{e}(G)$, the group $P_{7}$ acts fixed point freely on the set of elements of order 5 , and so $\left|P_{7}\right| \mid m_{5}=504$, which implies that $\left|P_{7}\right|=7$. Similarly, $\left|P_{5}\right|=5$.

We know that if $P$ and $Q$ are Sylow 7-subgroups of $G$, then $P$ and $Q$ are conjugate, which implies that $C_{G}(P)$ and $C_{G}(Q)$ are conjugate in $G$. Therefore $m_{21}=\phi(21) \cdot n_{7} \cdot k$, where $k$ is the number of cyclic subgroups of order 3 in $C_{G}\left(P_{7}\right)$. Since $n_{7}=m_{7} / \phi(7)=120,2 \times 720 \mid m_{21}$, which is a contradiction. Hence $21 \notin \pi_{e}(G)$. Similarly, $10 \notin \pi_{e}(G)$.

Since $21 \notin \pi_{e}(G)$, the group $P_{3}$ acts fixed point freely on the set of elements of order 7 , and $\left|P_{3}\right| \mid m_{7}$. Thus $\left|P_{3}\right| \mid 9$. Also since $10 \notin \pi_{e}(G),\left|P_{2}\right| \mid m_{5}=504$, and so $\left|P_{2}\right| \mid 2^{3}$.

If $\left|P_{3}\right|=3$, then $|G|=2^{m} \times 105$ and $m \leq 3$. On the other hand, $2520 \leq|G|$, a contradiction. Therefore $\left|P_{3}\right|=9$ and $|G|=2^{m} \times 315$ where $m \leq 3$. Since $2520 \leq|G|, m=3$ and then $|G|=2520=\left|A_{7}\right|$. By [4], since $A_{7}$ is a simple $K_{4}$-group, $G \cong A_{7}$.

Now suppose that $G$ be a group such that nse $(G)=\operatorname{nse}\left(A_{8}\right)=\{1,315$, $1232,1344,2688,3780,5040,5760\}$. First we prove that $\pi(G) \subseteq\{2,3,5$, $7\}$. Since $315 \in \operatorname{nse}(G)$, it follows that by $(*), 2 \in \pi(G)$ and $m_{2}=315$. Let $2 \neq p \in \pi(G)$, by $(*), p \mid\left(1+m_{p}\right)$ and $(p-1) \mid m_{p}$, which implies that $p \in\{3$, $5,7,19,2689,5041\}$.

If $19 \in \pi(G)$, then $m_{19}=3780$. On the other hand, by $(*)$ we conclude that if $38 \in \pi_{e}(G)$, then $m_{38} \in\{5760,3780,5040\}$ and $38 \mid\left(1+m_{2}+m_{19}+m_{38}\right)$, a contradiction. Therefore $38 \notin \pi_{e}(G)$. Thus the group $P_{19}$ acts fixed point freely on the set of elements of order 2 , and $\left|P_{19}\right| \mid m_{2}$, which is a contradiction. Hence $19 \notin \pi(G)$. Similar to the above discussion $2689,5041 \notin \pi(G)$, and so $\pi(G) \subseteq\{2,3,5,7\}$.

If 3,5 and $7 \in \pi(G)$, then $m_{3}=1232, m_{5}=1344$ and $m_{7}=5760$, by $(*)$. Also we can see easily that $G$ does not contain any elements of order 35,512 , $81,125,343$ and 768.

If $15,25,49 \in \pi_{e}(G)$, then $m_{15}=2688, m_{25}=3780$ and $m_{49}=1344$.

If $2^{a} \times 3^{b} \in \pi_{e}(G)$, then $1 \leq a \leq 6$ and $1 \leq b \leq 4$. 
If $2^{c} \times 5^{d} \in \pi_{e}(G)$, then $1 \leq c \leq 6$ and $1 \leq d \leq 2$.

If $2^{e} \times 7^{f} \in \pi_{e}(G)$, then $1 \leq e \leq 7$ and $1 \leq f \leq 2$. If $3^{k} \times 5^{h} \in \pi_{e}(G)$, then $1 \leq k \leq 3$ and $1 \leq h \leq 2$.

If $3^{l} \times 7^{m} \in \pi_{e}(G)$, then $1 \leq l \leq 3$ and $1 \leq m \leq 2$.

We show that $\pi(G)$ could not be the sets $\{2\},\{2,3\}$ and $\{2,3,5\}$ and $\{2$, $3,7\}$, and $\pi(G)$ must be equal to $\{2,3,5,7\}$.

Case a. If $\pi(G)=\{2\}$, then $\pi_{e}(G) \subseteq\left\{1,2,2^{2}, 2^{3}, 2^{4}, 2^{5}, 2^{6}, 2^{7}, 2^{8}\right\}$ and $|G|=2^{m}=20160+1232 k_{1}+1344 k_{2}+2688 k_{3}+3780 k_{4}+5040 k_{5}+5760 k_{6}$, where $m, k_{1}, k_{2}, k_{3}, k_{4}, k_{5}$ and $k_{6}$ are non-negative integers and $0 \leq k_{1}+k_{2}+$ $k_{3}+k_{4}+k_{5}+k_{6} \leq 1$. It easy to check that this equation has no solution.

Case b. We claim that $3 \in \pi(G)$. Suppose contrary, i.e, $3 \notin \pi(G)$. If 5 , $7 \notin \pi(G)$, then by Case a, we get a contradiction. Hence 5 or $7 \in \pi(G)$.

Let $5 \in \pi(G)$. Since $125 \notin \pi_{e}(G), \exp \left(P_{5}\right)=5$ or 25 .

If $\exp \left(P_{5}\right)=5$, then $\left|P_{5}\right| \mid\left(1+m_{5}\right)=1345$. Hence $\left|P_{5}\right|=5$, and so $n_{5}=1344 / 4|| G \mid$. Because $3 \notin \pi(G)$, we get a contradiction.

If $\exp \left(P_{5}\right)=25$, then $\left|P_{5}\right| \mid 125$. Suppose that $\left|P_{5}\right|=25$, then $n_{5}=$ $3780 / 20|| G \mid$, a contradiction. If $\left|P_{5}\right|=125$, then by Lemma $2.2,25 \mid m_{25}$, a contradiction. Thus $5 \notin \pi(G)$.

Let $7 \in \pi(G)$. Since $7^{3} \notin \pi_{e}(G), \exp \left(P_{7}\right)=7$ or 49 .

If $\exp \left(P_{7}\right)=7$, then $\left|P_{7}\right|=7$ and $n_{7}=m_{7} / 6=5760 / 6|| G \mid$, a contradiction.

If $\exp \left(P_{7}\right)=49$, then $\left|P_{7}\right|=49$. Hence $n_{7}=m_{49} / 42=32$ and by Sylow's theorem, we get a contradiction.

Case c. Let $\pi(G)=\{2,3\}$. Since $3^{4} \notin \pi_{e}(G), \exp \left(P_{3}\right)=3,3^{2}$ or $3^{3}$. If $\exp \left(P_{3}\right)=3$, then $\left|P_{3}\right| \mid\left(1+m_{3}\right)=1233$. Hence $\left|P_{3}\right| \mid 9$. Thus $\left|P_{3}\right|=3$ or 9. First suppose that $\left|P_{3}\right|=3$. Then $n_{3}=m_{3} / 2=1232 / 2|| G \mid$, a contradiction. Suppose that $\left|P_{3}\right|=9$. Thus $|G|=2^{m} \times 9=20160+1232 k_{1}+$ $1344 k_{2}+2688 k_{3}+3780 k_{4}+5040 k_{5}+5760 k_{6}$, where $m, k_{1}, k_{2}, k_{3}, k_{4}, k_{5}$ and $k_{6}$ are non-negative integers and $0 \leq k_{1}+k_{2}+k_{3}+k_{4}+k_{5}+k_{6} \leq 9$. It is clear that $|G| \leq 72000$. Since $20160 \leq|G| \leq 72000, m=12$. Therefore $16704=1232 k_{1}+1344 k_{2}+2688 k_{3}+3780 k_{4}+5040 k_{5}+5760 k_{6}$. By using an easy computer calculation, we can see that this equation has no solution.

Let $\exp \left(P_{3}\right)=9$. Since $m_{9} \in\{3780,5760\}$ and $\left|P_{3}\right| \mid\left(1+m_{3}+m_{9}\right)$, $\left|P_{3}\right|=9$. Hence $n_{3}=m_{9} / 6|| G \mid$, which is a contradiction.

Let $\exp \left(P_{3}\right)=27$. Since $m_{27} \in\{3780,5760\}$ and $\left|P_{3}\right| \mid\left(1+m_{3}+m_{9}+m_{27}\right)$, $\left|P_{3}\right| \mid$ 81. If $\left|P_{3}\right|=81$, then by Lemma $2.2,27 \mid m_{27}$, which is a contradiction. If $\left|P_{3}\right|=27$, then $n_{3}=m_{27} / 18|| G \mid$, a contradiction. 
Case d. Let $\pi(G)=\{2,3,5\}$. Since $5^{3} \notin \pi_{e}(G), \exp \left(P_{5}\right)=5$ or 25 . If $\exp \left(P_{5}\right)=5$, then $\left|P_{5}\right| \mid\left(1+m_{5}\right)=1345$. Hence $\left|P_{5}\right|=5$, and $n_{5}=1344 / 4 \mid$ $|G|$, a contradiction. If $\exp \left(P_{5}\right)=25$, then $\left|P_{5}\right| \mid\left(1+m_{5}+m_{25}\right)$. Thus $\left|P_{5}\right| \mid 125$ and $\left|P_{5}\right|=25$ or 125 . If $\left|P_{5}\right|=25$, then $n_{5}=3780 / 20|| G \mid$, a contradiction. If $\left|P_{5}\right|=125$, then $25 \mid m_{25}$, a contradiction.

Case e. Let $\pi(G)=\{2,3,7\}$. Since $7^{3} \notin \pi_{e}(G), \exp \left(P_{7}\right)=7$ or 49 . If $\underline{\exp \left(P_{7}\right)}=7$, then $\left|P_{7}\right| \mid\left(1+m_{7}\right)$. Thus $\left|P_{7}\right|=7$ and $n_{7}=960$. Since $n_{7}|| G \mid$ and $5 \notin \pi(G)$, we get a contradiction.

If $\exp \left(P_{7}\right)=49$, then $\left|P_{7}\right| \mid\left(1+m_{7}+m_{49}\right)$. Thus $\left|P_{7}\right|=49$ and $n_{7}=32$. By Sylow's theorem, we get a contradiction.

Case f. Let $\pi(G)=\{2,3,5,7\}$. Since $35 \notin \pi_{e}(G)$, the group $P_{7}$ acts fixed point freely on the set of elements of order 5 , and so $\left|P_{7}\right| \mid m_{5}=1344$, which implies that $\left|P_{7}\right|=7$. Similarly, we can conclude that $\left|P_{5}\right|=5$. We have $m_{21}=\phi(21) \cdot n_{7} \cdot k$, where $k$ is the number of cyclic subgroups of order 3 in $C_{G}\left(P_{7}\right)$. Since $n_{7}=m_{7} / \phi(7)=960,2 \times 5760 \mid m_{21}$, a contradiction. Hence $21 \notin \pi_{e}(G)$. Similarly, $10 \notin \pi_{e}(G)$.

Since $21 \notin \pi_{e}(G)$, the group $P_{3}$ acts fixed point freely on the set of elements of order 7. Then $\left|P_{3}\right| \mid m_{7}=5760$. Thus $\left|P_{3}\right| \mid 9$. Also since $10 \notin \pi_{e}(G)$, $\left|P_{2}\right| \mid m_{5}=1344$, and so $\left|P_{2}\right| \mid 2^{6}$. If $\left|P_{3}\right|=3$, then $|G|=2^{m} \times 105$. On the other hand, since $\left|P_{2}\right| \mid 2^{6}, m \leq 6$. Since $|G| \leq 20160,2^{m} \times 105 \leq 20160$, a contradiction. Therefore $\left|P_{3}\right|=9$ and then $|G|=\left|A_{8}\right|$. By [4], since $A_{8}$ is a simple $K_{4}$-group, $G \cong A_{8}$, and the proof is complete.

\section{Acknowledgment}

The authors is thankful to the referee for carefully reading the paper and for his valuable suggestions. Partial support by the Center of Excellence of Algebraic Hyperstructures and its Applications of Tarbiat Modares University (CEAHA) is gratefully acknowledge by the third author (AI).

\section{References}

[1] A. K. Asboei and A. Iranmanesh, A characterization of Linear group $L_{2}(p)$, Czechoslovak Math. J (In press).

[2] A. K. Asboei, S. S. S. Amiri, A. Iranmanesh and A. Tehranian, A characterization of Symmetric group $S_{r}$, where $r$ is prime number, Ann. Math. Inform, 40, 2012, 13-23. 
[3] A. K. Asboei, S. S. S. Amiri, A. Iranmanesh and A. Tehranian, A new characterization of sporadic groups by NSE and order, J. Algebra Appl, 12(2), 2013, 1-3.

[4] C. G. Shao, W. Shi and Q. H. Jiang, Characterization of simple $K_{4}$-groups, Front Math China, 3, (2008), 355-370.

[5] G. Frobenius, Verallgemeinerung des sylowschen satze, Berliner sitz, (1895), 981-993.

[6] G. Miller, Addition to a theorem due to Frobenius, Bull. Am. Math. Soc, 11, (1904), 6-7.

[7] J. H. Conway, R. Curtis, S. Norton and et al, Atlas of Finite Groups, Clarendon, Oxford, 1985.

[8] M. Khatami, B. Khosravi and Z. Akhlaghi, A new characterization for some linear groups, Monatsh Math, 163, (2011), 39-50.

[9] R. Shen, C. G. Shao, Q. Jiang, W. Shi and V. Mazurov, A New Characterization of $A_{5}$, Monatsh Math, 160, (2010), 337-341.

[10] V. D. Mazurov and E. I. Khukhro, Unsolved Problems in group theory: the Kourovka Notebook, 16 ed, Novosibirsk, Inst. Mat. Sibirsk. Otdel. Akad, 2006.

Alireza Khalili Asboei,

Department of Mathematics,

Science and Research Branch,

Islamic Azad University, Tehran, Iran.

Email: khaliliasbo@yahoo.com

Syyed Sadegh Salehi,

Department of Mathematics,

Science and Research Branch,

Islamic Azad University, Tehran, Iran.

Email: salehissss@yahoo.com

Ali Iranmanesh,

Department of Mathematics,

Tarbiat Modares University

P. O. Box: 14115-137, Tehran, Iran.

Email: iranmanesh@modares.ac.ir

Abolfazl Tehranian,

Department of Mathematics,

Science and Research Branch,

Islamic Azad University, Tehran, Iran.

Email: tehranian1340@yahoo.com 